\title{
Intra-retinal Layer Segmentation of Macular OCT Images using Edge Superpixels and Manifold Ranking Method
}

\author{
Zhijun $\mathrm{Gao}^{1,2}$, Wei Bu ${ }^{3, *}$, Xiangqian $\mathrm{Wu}^{1}$ and Yalin Zheng ${ }^{4}$ \\ ${ }^{1}$ School of Computer Science and Technology, Harbin Institute of Technology, \\ Harbin 150001, China \\ ${ }^{2}$ College of Computer and Information Engineering, Heilongjiang University of \\ Science and Technology, Harbin 150022, China \\ ${ }^{3}$ Dept of Media Technology \& Art, Harbin Institute of Technology, Harbin \\ 150001, China \\ ${ }^{4}$ Department of Eye and Vision Science, Institute of Ageing and Chronic Disease, \\ University of Liverpool, UCD Building, Liverpool L69 3GA, United Kingdom \\ buwei@hit.edu.cn
}

\begin{abstract}
In order to effectively segment the eleven surfaces of ten retinal layers in optical coherence tomography (OCT) images, in this paper, a novel automated intra-retinal layer segmentation method based on the edge superpixel and manifold ranking method was proposed as illustrated in three steps. Firstly, we defined the edge superpixel that can well overcome some disturbances from the intrinsic speckle noise and organic texture artifacts, and designed a simple adaptive filter that wass able to automatically remove some disturbances from vitreous artifacts. Secondly, the OCT image was represented as a weighted graph with edge superpixels as nodes, and the affinity matrix were computed. Finally, each node was ranked optimally with the texture and spatial cues using the graph-based manifold ranking technology, so that the eleven surfaces of ten retinal layers in optical coherence tomography images were exactly, fast and reliably quantified in two stages. The proposed algorithm was evaluated by OCT images from two different databases, and was compared with the manual tracings of two independent observers and the Iowa algorithm. The experiment demonstrated the promising results in term of the mean unsigned boundaries errors and the mean signed boundaries errors, and the proposed algorithm outperformed the Iowa algorithm around macular fovea. In addition, the proposed algorithm could effectively segment the eleven surfaces of ten retinal layers in optical coherence tomography images with the central serous retinopathy (CSR).
\end{abstract}

Keywords: Optical coherence tomography; Layer segmentation; Edge superpixel; Manifold ranking

\section{Introduction}

Optical coherence tomography was first introduced by Huang et al. [1], and it is a powerful, noninvasive and high resolution imaging modality, compared with the ocular fundus photography and fundus fluorescein angiography. Particularly, with the recent advancement of this technology from time domain to spectral domain optical coherence tomography (SD-OCT) [2], currently, the OCT has been widely used in the diagnosis and assessment of a variety of ocular diseases such as diabetic retinopathy, glaucoma and CSR [3-5]. However, lacking fast and accurate quantification approach for more 3D OCT data, it is unwelcome for clinicians or ophthalmologists to diagnose directly for retinal diseases by calculating nerve fiber layer thickness, or inner plexiform layer thickness, or total retinal thickness. Therefore, it has been actively explored for OCT image 
segmentation during the last two decades, and a number of retinal layer segmentation algorithms of OCT images data have been proposed to improve the clinical benefit of the OCT technology. Based on the dimension (D) of the image analysis, depending on the exploration of the single pixel's intensity and gradient information, the OCT segmentation algorithms mainly concentrated on the two categories of the single pixel and superpixel as follows.

The early single pixel method used a 1-D edge detection kernel approach to measure total retinal thickness. Koozekanani et al., employed a Markov random field (MRF) model to detect robustly the inner and outer retinal boundaries for macula OCT images [6].

Then, Mishra et al. also proposed a modified active contour algorithm that achieved accurate intra-retinal segmentation on low contrast image [7]. Compared to the initial Ascan methods, though the active contour algorithm showed good performance in resistance to $2 \mathrm{D}$ noise and in error, there still exists the limitation of selecting the initial seed points of the contour. Pattern recognition techniques have increasingly played an important role in medical image segmentation, recently, and a number of unsupervised and supervised pattern recognition techniques have explored for the retinal layers segmentation. Mayer et al. used an unsupervised technique based on a fuzzy C-means clustering method to segment RNFL [8]. Vermeer et al. also presented a support vector machine (SVM) classifiers that were able to segment six retinal layers [9], a major drawback was that more time was required to complete the segmentation task.

With the application of the graph optimal method for medical image segmentation, graph search technique and graph cuts technique were successively proposed for retinal layers segmentation. Haecker et al. firstly performed a complex 3D graph search method that successfully extracted two intra-retinal layers [10]. Based on the graph search method, combining with regional information, a multilayer segmentation method based on the graph cuts was proposed by Garvin et al., and the method demonstrated superior results for high quality 3D OCT data [11]. However, the method needs much more complex constraints that also lead to the high computation time, and a major flaw was a less ideal foveal detection. Recently, Shi and Chen et al. successfully used a multiresolution graph search based surface detection method that can automatically segment the retinal layers in 3-D OCT data with serous retinal pigment epithelial detachments [12]. Besides, the dynamic programming technique emerged as one of the important retinal layers segmentation method. Chiu et al. used a dynamic programming technique that was able to extract eight retinal boundaries [13], and Yang et al. also introduced a dual-scale gradient information model to segment eight retinal layers [14]. Han et al. also proposed an intelligent tracking kernel method to segment nine boundaries of eight retinal layers [15], but its processing time was also relatively high.

Recently, Kafieh et al., presented a coarse grained diffusion map method that can segment eleven retinal layers [16], the method likes superpixel approaches that can avoid noise in OCT images. However, it still needs indirectly locate all the boundaries by single pixels, and the coarse graining computation, which result in the high time-consuming. Most existing retinal layers segmentation algorithms mainly focus on the single pixel or region based on its intensity or gradient within a local context. The single pixel can't greatly reduce the complexity of image processing tasks, and is relatively susceptible to speckle noise or artifact. Particularly, for the large 3D OCT images, it will be inconvenient for ophthalmologists or clinicians to diagnose.

Inspired by superpixel, a graph-based SLIC superpixels and manifold ranking algorithm was well focused on the segmentation of the OCT images [17]. Using the superpixels and connected components, the novel method can't only effectively avoid the speckle noise or artifact, but also fast and exactly segment the eleven surfaces and ten layers. However, there exist also two flaws or limitations in the method. On the one hand, in order to avoid the disturbance of the artifact, the number of superpixels need be set 
beforehand by artificial means. On the other hand, in order to locate the connected components of the boundaries ILM and IS/CL, the Dijkstra's algorithm was utilized, and a morphological closing was performed on the two paths with a disk structuring element, the step needs not only select a disk structuring element with the right size, but also their implementation need a certain processing times.

This work is motivated by the parameter reduction, speed up, and the exact boundaries detection. A novel edge superpixel-based manifold ranking approach focused on intraretinal layer segmentation of OCT images, since its eleven intra-retinal boundaries mainly correspond to high, middle or low contrast in pixels intensity, positive or negative vertical gradient values, and their spatial relationship between intra-retinal boundaries. Fig.1 illustrates eleven intra-retinal boundaries and surfaces we desired to find in macular spectral-domain OCT images.

In comparision with the single methods and SLIC superpixels method [17], contributions in this paper mainly include as follows: 1) A simple adaptive filter was designed, and could well replace the superpixel and the Dijkstra's algorithm to effectively remove some disturbances from vitreous artifacts. 2) The edge superpixel was defined, and was able to robustly extract the eleven boundaries around macular fovea. 3) The proposed method optimized the boundaries detection of the three stages into two stages. 4) A novel edge superpixel-based manifold ranking approach can detect the eleven boundaries of the macular OCT images with CSR, the errors were not only low, the detection was also fast and robust.

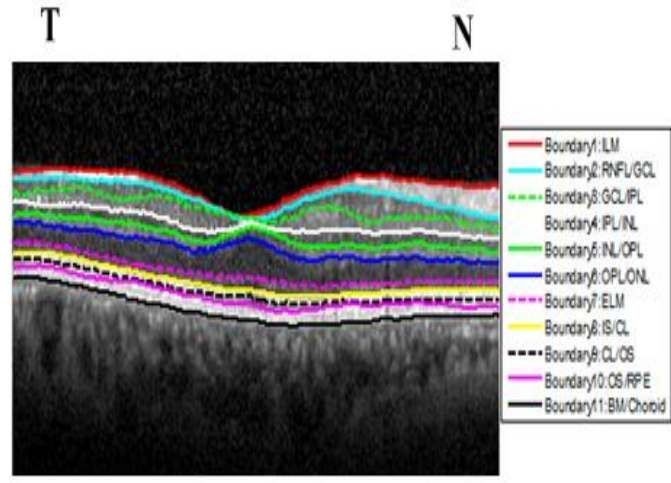

(a)

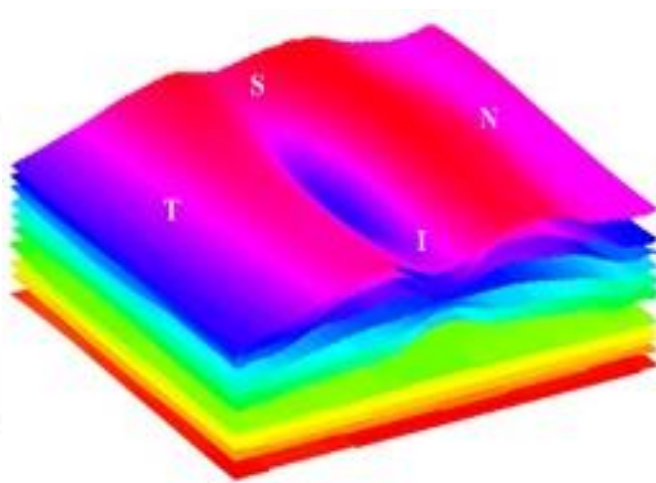

(b)

Figure 1. Illustrates Segmentation Results of Eleven Intra-Retinal Surfaces with Ten Retinal Layers. (a) Eleven intra-Retinal Boundaries of 2D slice image from top to bottom: boundary 1 ILM, boundary 2 NFL/GCL, boundary 3 GCL / IPL, boundary 4 IPL/INL, boundary 5 INL/OPL, boundary 6 OPL/ONL, boundary 7 ELM, boundary $8 \mathrm{IS} / \mathrm{CL}$, boundary $9 \mathrm{CL} / \mathrm{OS}$, boundary 10 OS/RPE, and boundary $11 \mathrm{BM} /$ Choroid. (b) The Segmented Surfaces of 3D OCT image (T: temporal; I: inferior; N: nasal; S: superior).

Under fewer parameters and faster time, the research demonstrated that such an optimal approach was able to automatically segment eleven boundaries of ten retinal layers in OCT images, and improve the accuracy, efficiency, and robustness of retinal layers segmentation.

The rest of this paper is organized as follows, Section 2 defines the edge superpixel, briefly introduces the construction of the weighted graph and the manifold ranking method, and describes detailly the proposed intra-retinal layers segmentation algorithm using edge superpixel and graph-based manifold ranking technology. Section 3 presented the experiments and results. Finally, Section 4 concludes the paper. 


\section{Material and Methods}

\subsection{Edge Superpixel}

Superpixels are some meaningful atomic regions by dividing the whole pixels. Not only they can retain the rigid structure of the pixel grid, but also extract image redundancy and greatly reduce the complexity of subsequent image processing tasks. The superpixels are also becoming increasingly popular in computer vision applications, such as object detection [18] and saliency detection [19].

In this work, inspired by the theory of the vertex coloring and edge coloring in graph theory [20], the edge superpixel was defined by the connected components, and makes the boundary detection easier to understand and analyze. It is shown as Figure 2e that fourteen edge superpixels can be detected by a classic canny edge detector [21]. And the positive edge superpixel was defined by the edge superpixel whose vertical gradient value is positive, conversely, and was called the negative edge superpixel. They are some perfect examples that the boundaries $1,3,5,7,8$, and 10 all correspond to the positive edge superpixels as shown the edges in Fig ure $2 \mathrm{c}$ and the blue and red edges in Figure $3 \mathrm{k}$, and the other three boundaries correspond to the negative edge superpixels as shown the other color edges in Figure.3k.

\subsection{Weighted Graph Construction and Affinity Matrix}

It is shown as the Figure 2e, a weighted graph $G=(V, E, W)$ is constructed by the edge superpixels, well represents OCT image, and exploit the texture information and the spatial relationship. Where each node corresponds to an edge superpixels. The edge set $E$ is a set of undirected edges, and $W$ denotes the affinity matrix that represents the weights of the edges between two arbitrary nodes. In this work, each node is not only connected to those nodes neighboring it, but also connected to the nodes with its neighboring node, such as the two blue nodes as shown in Figure 2e. It is clear that the weighted graph $G$ is a sparse. That is, the affinity matrix $W$ is sparse matrix, which is also able to upgrade compute rate. In this work, $W$ is defined by Eqs.1, due to eleven intra-retinal boundaries of the OCT image mainly correspond to low, middle or high contrast in pixels intensity, positive or negative vertical gradient values, and their spatial relationship between intraretinal boundaries.

$$
w(i, j)=\exp \left(\frac{\left\|F_{i}-F_{j}\right\|_{2}^{2}}{-2 \delta^{2}}\right) * \operatorname{sgn}\left(X_{i}-Y_{j}-e_{1}\right) * \operatorname{sgn}\left(e_{2}-\left(X_{i}-Y_{j}\right)\right)
$$

Where $F_{i}=\left(\tilde{f}_{i}, \tilde{g}_{i}\right)$ or $\left(\tilde{g}_{i}, \bar{y}_{i}\right), \tilde{f}_{i}$ is the sum of the edge superpixel corresponding to a node in the intensity of the pixels, $\tilde{g}_{i}$ denotes the sum of the edge superpixel corresponding to a node in the gradient value of the pixels, $\bar{y}_{i}$ is the mean of the edge superpixel corresponding to a node in the row-coordinate of the pixels, $X_{i}$ and $Y_{i}$ denote respectively the start and end vertex of edge superpixel corresponding to a node in the coordinate of the pixels, for $i, j=1,2, \ldots,|V|$. In Eq.1, naturally, texture information could be captured by the first term, and spatial relationship information by the second term. That is, the closer their intensity value or spatial distance, the higher the affinity value $W(i, j)$ between nodes. So that Eq.1 can represent texture information and constraint spatial relationship well. 


\subsection{Manifold Ranking}

The manifold ranking method is used to exploit the intrinsic manifold structure of data for labeled graph [22], essentially, can be equivalent to a one-class classification problem [23], and has achieved great success on image saliency detection [19]. Generally, for a dataset $X=\left\{x_{1}, x_{2}, \cdots, x_{n}\right\} \in \square^{m^{*} n}$, and a weighted graph $G=(V, E, W)$ is constructed for the dataset $X$, where the nodes set $V$ are the same to the dataset $X$, the edges set $E=\left\{e_{i j} \mid\right.$ $\left.e_{i j}=\left(x_{i}, x_{j}\right), i, j=1,2, \ldots, n\right\}$ and the affinity matrix $W=\left[w_{i j}\right]_{n \times n}$. Thereby, the degree matrix $D$ is equal to $\operatorname{diag}\left\{d_{11}, d_{22}, \ldots, d_{n n}\right\}$, where $d_{i i}=\sum_{j} w_{i j}$. Then, similar to the spectral clustering algorithms [24], the optimal ranking problem can be computed by the Eq.2. Certainly, the optimal ranking solution could be conveniently written as by Eq. 3 using the unnormalized Laplacian matrix.

$$
f^{*}=\arg \min _{f} \frac{1}{2}\left(\sum_{i, j=1}^{n} w_{i j}\left\|\frac{f_{i}}{\sqrt{d_{i i}}}-\frac{f_{j}}{\sqrt{d_{j j}}}\right\|^{2}+\mu \sum_{i=1}^{n}\left\|f_{i}-y_{i}\right\|\right),
$$

Where the first term represents the smoothness constraint, that is, if both the vertices $x_{i}$ and $x_{j}$ are adjacent, then their rank scores $f_{i}$ and $f_{j}$ should not change too much. The second term is the fitting constraint, that is, $f_{i}$ should not differ too much from the initial query assignment $y_{i}$. The parameter $\mu$ can well control their balance.

$$
f^{*}=(D-\theta W)^{-1} y
$$

Where the vector $y$ consists of zeros and some queries that are assign a positive ranking score (such as 1), and zero to the remaining points.

\subsection{Boundaries Detection by Two-stage}

In this section, the proposed two-stage scheme is utilized for OCT boundaries detection using edge superpixels and graph-based manifold ranking method with texture information, the spatial relationship.

2.4.1 Detect the two prominent boundaries: The ILM and IS/CL are the two most prominent boundaries among eleven boundaries in an SDOCT retinal image, due to their high contrast in pixel intensity. Thereby, both ILM and IS/CL boundaries are firstly extracted by the proposed algorithm as follows. Inevitably, it is possible that some intrinsic speckle noise and vitreous artifacts make disturbance to exactly extract their endpoints, for example, in the upper left and upper right corner of Figure 2a, there exist two vitreous artifacts.

Firstly, in order to simultaneously reduce noise and preserve edges, a classic and effective median filter is quickly performed in OCT images as shown in Figure $2 b$. Subsequently, a classic canny edge detector is used to detect the high contrast edge superpixels by high threshold, so that the significant boundaries could be not only detected, but also could remove the false boundaries as shown in Figure 2c. The high threshold value is equal to the half of the automated threshold. For the possible presence of two vitreous artifacts, which easily joint with ILM boundary in Figure 2c. Using the spatial cues of the endpoints with the edge superpixels, an adaptive filter was designed to automatically cut the joint between the true boundary and the false boundary. Namely, for the left region of the macular center, if the right endpoint of one edge superpixels is adjacent with the other edge superpixels above it, then the latter will be cut in the right endpoint of the former. Conversely, for the right region of the macular center, if the left endpoint of one edge superpixels is adjacent with the other edge superpixels above it, then 
the latter will be cut in the left endpoint of the former. The yellow edge of the vitreous artifact is effectively cut by the adaptive filter as shown in Figure 2d. Next, a weighted graph $G=(V, E, W)$ is constructed by the edge superpixels, and its affinity matrix $W$ is computed by Eq.1, where the $F_{i}=\left(\tilde{f}_{i}, \tilde{g}_{i}\right)$. Then, all nodes are ranked according to their final ranking scores by Eq.3. Where the queries are automatically selected two nodes from the highest gradient value of the nodes as shown in Figure $2 \mathrm{f}$, so that the queries of both ILM (up) and IS/CL (down) could be selected. Figure $2 \mathrm{~g}$ shows the result of the edge superpixels with manifold ranking, which could not only well reserve the edge superpixels both ILM and IS/CL, but also effectively reject some edge superpixels of the other salient noise boundaries relative to the result of Figure 2d. Finally, in order to show so much smooth boundary, respectively, the results are enhanced by sixteen and twenty orders polynomial smoothing as shown in Figure $2 \mathrm{~h}$. Meanwhile, the other edge superpixels can be restricted to successively two smaller search areas as shown in Figure $2 \mathrm{i}$, respectively, all the other boundaries are detected as follows.

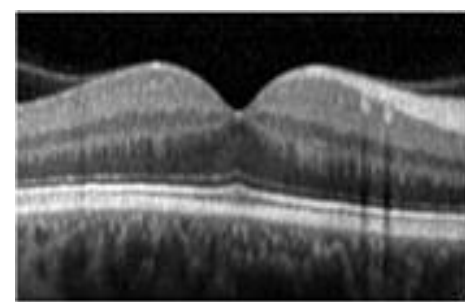

(a)

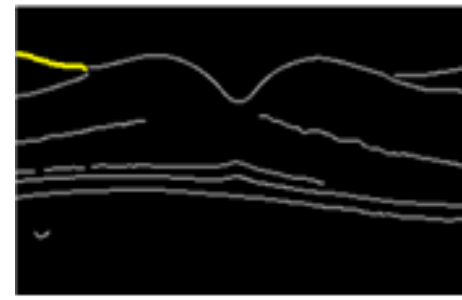

(d)

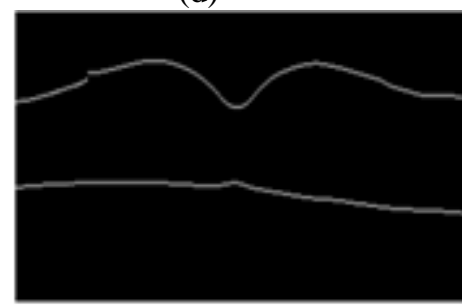

(g)

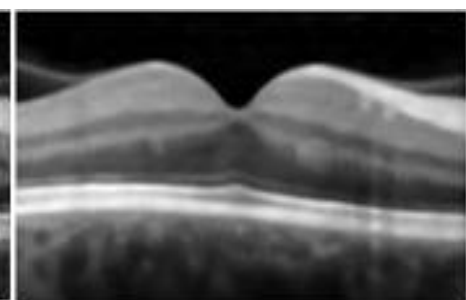

(b)

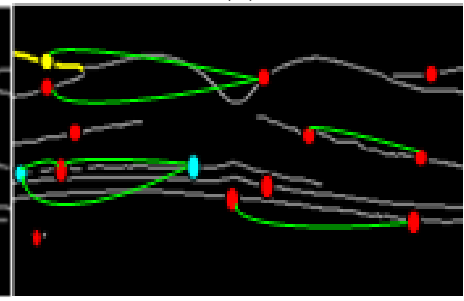

(e)

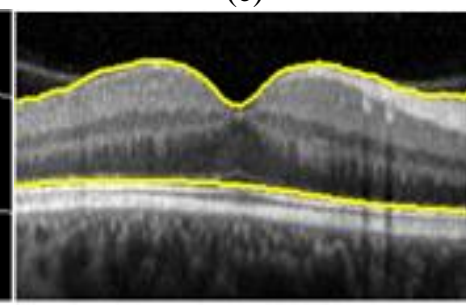

(h)

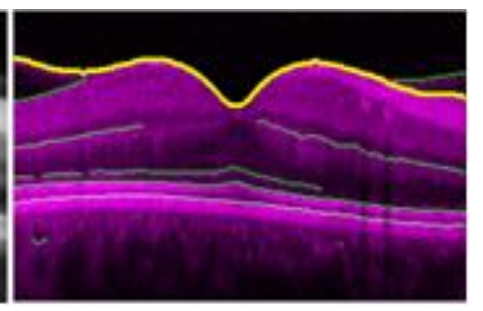

(c)

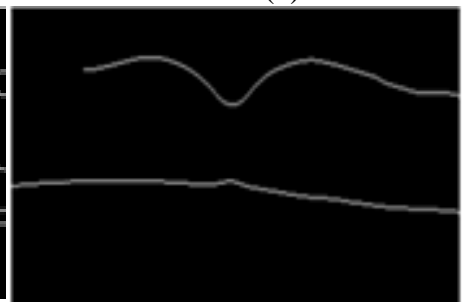

(f)

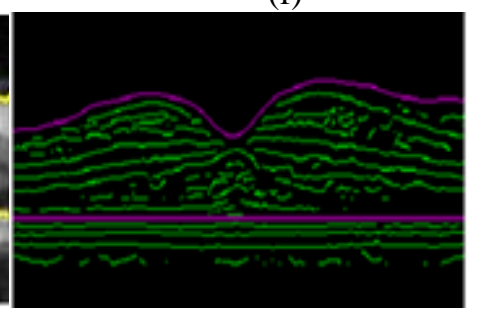

(i)

Figure 2. Images show how the ILM and IS/CL boundaries are found via the graph-based manifold ranking approach. (a)Original OCT image. (b) Result of applying median filter.(c) High contrast edge superpixels.(d) Result of applying an adaptive filter. (e) Our graph mode (f) Automatically selected queries the ELM and IS/CL boundaries. (g) Detected the edge superpixels of both ILM and IS/CL boundaries by manifold ranking method. (h) Result of the ILM and IS/CL boundaries after smoothing. (i) The main edge superpixels

2.4.2 Detect the other weak boundaries: In order to the next segmentation step in the smaller image sizes and more consistent shape, and make visualization easier and conform to clinical practice [12], firstly, the OCT image is aligned according to the boundary IS/CL. Then, the low and middle contrast edge superpixels can be also detected in the aligned image by a classic canny edge detector with low-valued 
threshold $\left(0.05^{*}\right.$ automated threshold $)$, which detects the significant boundaries and preserves other potential boundaries as shown in the Fig. 2i. During the remaining nine boundaries, in the spatial relationship, the boundaries 2, 3, 4, 5, and 6 are between the boundary ILM and boundary IS/CL, and the boundaries 7, 9, 10 and 11 are around the boundary IS/CL. In the texture relationship, due to the contrast of the boundary 5 is higher than the contrast of the boundary 3 , the detection of the boundary 5 is easily compared to the boundary 3, though they all correspond to the positive edge superpixels. Similarly, the detection of the boundaries 4 and 6 are easily compared to the boundary 2 , though they all correspond to the negative edge superpixels. Both the detection of the boundaries 7 and 10 all correspond to the positive edge superpixels, conversely, both the detection of the boundaries 9 and 11 all correspond to the negative edge superpixels, their contrasts are similar each other. Therefore, the boundaries 5, 4, 6, 2, 3, 7, 9, 10 and 11 could be successively detected by manifold ranking method as follows.

Next, successively, we could construct the weighted subgraphs $G_{5}=\left(V_{5}, E_{5}, W_{5}\right)$, $G_{3}=\left(V_{3}, E_{3}, W_{3}\right) G_{7}=\left(V_{7}, E_{7}, W_{7}\right)$ and $G_{10}=\left(V_{10}, E_{10}, W_{10}\right)$ by the positive edge superpixels, and $G_{4}=\left(V_{4}, E_{4}, W_{4}\right), G_{6}=\left(V_{6}, E_{6}, W_{6}\right), G_{2}=\left(V_{2}, E_{2}, W_{2}\right), G_{9}=\left(V_{9}, E_{9}, W_{9}\right), G_{11}=\left(V_{11}, E_{11}\right.$, $\left.W_{11}\right)$ by the negative edge superpixels. And then their affinity matrices $W_{5}, W_{4}, W_{6}, W_{2}$, $W_{3}, W_{7}, W_{9}, W_{10}$, and $W_{11}$ are also computed successively by Eq.4 and Eq.1. Where $F_{i}=\left(\tilde{g}_{i}, \bar{y}_{i}\right)$ in Eq.1, whose purposes are able to constraint spatial and texture relationship between nodes well.

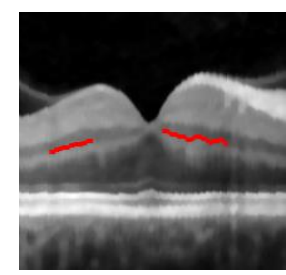

(a)

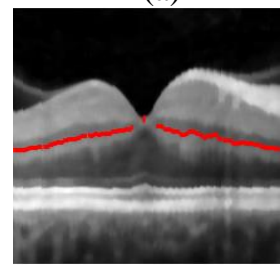

(f)

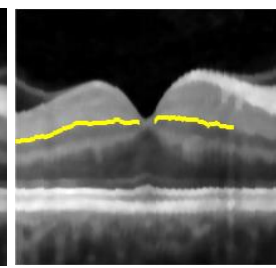

(b)

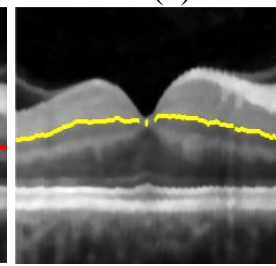

(g)

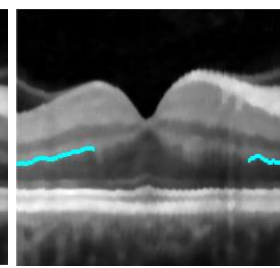

(c)

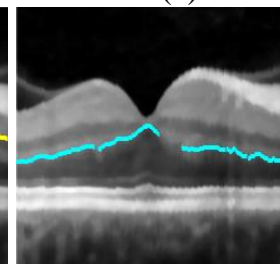

(h)

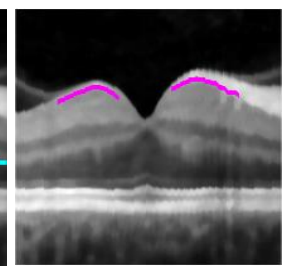

(d)

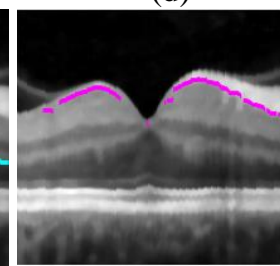

(i)

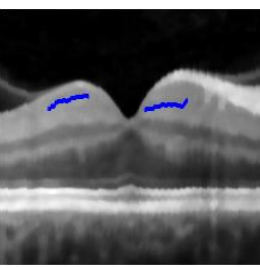

(e)

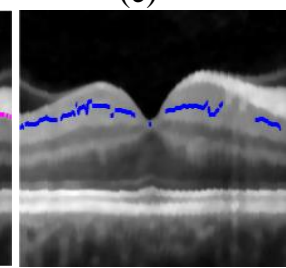

$(\mathrm{g})$

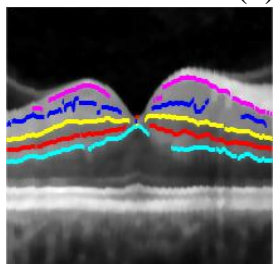

(k)

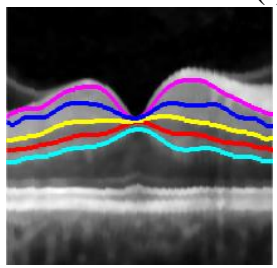

(1)

Figure 3. Images show how the boundaries between ILM and IS/CL are found via the graph-based manifold ranking approach. (a)-(e)The queries of the boundary 5 INL/OPL, boundary 4 IPL/INL, boundary 6 OPL/ONL, boundary 2 NFL/GCL, and boundary 3 GCL/IPL, respectively. (f)-(j) Result of the edge superpixels of the boundary 5 INL/OPL, boundary 4 IPL/INL, boundary 6 OPL/ONL, boundary 2 NFL/GCL and boundary 3 GCL/IPL with manifold ranking, respectively. (k) Ranked the edge superpixels between boundaries 2 and 6. (l) Result of boundaries 2, 3, 4, 5 and 6 after smoothing. 


$$
W_{k}(i, j)=W(i, j) \cdot * \operatorname{sgn}\left(s_{k} * W_{G}(i)\right) * * \operatorname{sgn}\left(W_{R}(i)-\left(b d_{k 1}\left(\bar{x}_{i}\right)+d_{k 1}\right)\right) \cdot * \operatorname{sgn}\left(b d_{k 2}\left(\bar{x}_{i}\right)+d_{k 2}-W_{R}(i)\right),
$$

Where $W_{G}(i)=\tilde{g}_{i}, W_{R}(i)=\bar{y}_{i}, i=1,2, \ldots,|V|$, and $k=2,3,4,5,6,7,9,10$, and 11. In the Eq.4, the first term $W(i, j)$ is from Eq.1. The second term $\operatorname{sgn}\left(s_{k} * W_{G}(i)\right)$ presents the positive or negative edge superpixels, and the variable $s_{k}$ is 1 when the node represents the positive edge superpixel, conversely, $s_{k}$ is -1 . The third and fourth terms are the spatial constraint. For the $k$ th boundary, both $b d_{k 1}\left(\bar{x}_{i}\right)+d_{k 1}$ and $b d_{k 2}\left(\bar{x}_{i}\right)+d_{k 2}$ represent the upper and lower bound of the $i$ th node, namely, the product of both the third and fourth terms is 1 if the $i$ th node belongs to the corresponding region, otherwise, it is zero. Where $\bar{x}_{i}$ denotes the mean of edge superpixels corresponding to the $i$ th node in the columncoordinate of the pixels. Therefore, in neighboring regions, the $W_{k}(i, j)$ is a positive value when both two nodes are positive or negative edge superpixels, conversely, $W_{k}(i, j)$ is 0 . So that Eq.4 can present texture information and constraint spatial relationship well.

For that reason, in Eq.4, the $s_{5}, s_{3} s_{7}$ and $s_{10}$ are equal to 1 , and conversely the other $s_{i}$ is equal to -1 . In Eq.4, let $d y_{i j}$ denote the mean distance of the rowcoordinate of the $i$ th boundary and the $j$ th boundary. For $k=5$, let both $b d_{k 1}$ and $b d_{k 2}$ respectively correspond to the boundary 1 and boundary 8 , so $d_{51}$ and $d_{52}$ are respectively equal to $0.1 * d y_{18}$ and $-0.25 * d y_{18}$. For $k=4$ and 6 , both $b d_{41}$ and $b d_{42}$ all correspond to the boundary 5 , both $b d_{61}$ and $b d_{62}$ respectively correspond to the boundary 5 and boundary $8, d_{41}$ is equal to $-0.25^{*} d y_{15}, d_{42}$ is equal to $-1, d_{61}$ is equal to 1 , and $d_{62}$ is equal to -12 . For $k=2$, both $b d_{21}$ and $b d_{22}$ respectively correspond to the boundaries 1 and $4, d_{21}$ is equal to 1 , and $d_{22}$ is equal to $-0.3 * d y_{14}$. For $k=3$, $b d_{31}$ and $b d_{32}$ respectively correspond to the boundary 2 and the boundary $4, d_{31}$ and $d_{32}$ are respectively equal to 1 and -1 . Finally, for $k=7,9,10$ and 11 , let both $b d_{k 1}$ and $b d_{k 2}$ correspond to the boundary $8, d_{71}$ and $d_{72}$ be respectively -10 and -1 , and conversely $d_{91}=1, d_{92}=10, d_{101}=3, d_{102}=20, d_{111}=10$, and $d_{112}=25$.

Then, successively, the nodes of the nine affinity matrices are ranked according to their final ranking scores by Eq.3. For the 5 th and $3 r d$ boundaries, respectively, their queries are automatically selected two nodes from the highest gradient value of the nodes for the left and right parts of the own boundary as shown in Fig. 3a and 3e, which could avoid no connectivity of the boundary due to its low contrast in pixel intensity around macular fovea. Conversely, For the 6 th, 4 th and $2 n d$ boundaries, respectively, their queries are automatically selected two nodes from the lowest gradient value of the nodes for the left and right parts of the own boundary as shown in Fig. 3b, 3c and3d. Fig. 3f-3j show the results of the edge superpixels that are optimally ranked by manifold ranking method, from left to right, and belong to the boundaries $5,4,6,2$ and 3 . By contrast, due to the boundaries 7, 9, 10 and 11 are barely affected by macular fovea. For the 7 th and 10th boundaries, respectively, their queries are automatically selected one node from the highest gradient value of the nodes. Conversely, for the 9th and 11th boundaries, respectively, their queries are automatically selected one node from the lowest gradient value of the nodes. Fig. 4a shows the four edge superpixels are successively belong to queries of the boundaries 7, 9, 10 and 11 from up to down. Fig. 4b shows the results of the edge superpixels that are optimally ranked by manifold ranking method, from up to down, and belong to the boundaries 7, 9, 10 and 11. The ranking results could not only effectively reject some edge superpixels of the other salient noise boundaries, but also well reserve the edge superpixels of the five and four boundaries as shown in the Fig. 3k and Fig. 4b, relative to the Fig. 2i. Particularly, in Fig. 4b, such as the right end of the boundary 7 can't be detected, since its low or middle contrast in pixel intensity. Nevertheless, in order to the next smoothing step, the right end of the boundary 7 is defined to the mean vertical distance of the edge superpixels between the detected boundary 7 and 8 . 
Finally, in order to show so much smooth boundary, for the boundaries 5, 4, 6, 2, 3, 7, 9, 10 and 11, respectively, the results are enhanced by sixteen, sixteen, sixteen, twenty, sixteen, twenty, six, six and six orders polynomial smoothing as shown in Fig. 31 and Fig. 4c. And Fig.4d shows the extracted results of the eleven boundaries.

The eleven surfaces form the final segmentation on 3D OCT as shown in Fig.1b.

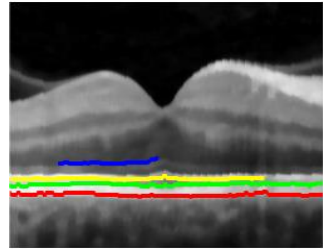

(a)

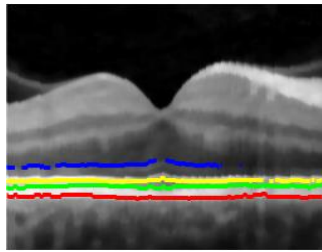

(b)

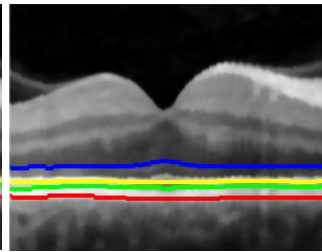

(c)

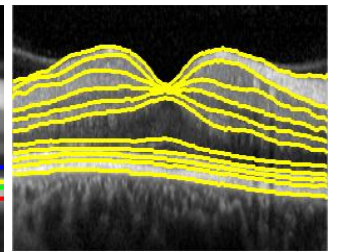

(d)

Figure 4. Images show how the ELM and boundaries below IS/CL are found via the graph-based manifold ranking approach. (a) Automatically selected queries of the ELM and boundaries below IS/CL. (b) Result of the edge superpixels of the ELM and boundaries below IS/CL with manifold ranking approach. (c) Result of boundaries 7, 9, 10 and 11 after smoothing. (d) Final segmentation for the original image after smoothing.

The main steps of the proposed lay segmentation algorithm are summarized in Algorithm 1.

\section{Algorithm 1}

Input: An OCT image and required parameters

Step1. Detect the two prominent boundaries.

Step 1.1 Detect the high contrast edge superpixels by canny edge detector for filtered image.

Step 1.2 Perform an adaptive filter on edge superpixels image, construct a graph $G$ with edge superpixels as nodes, and compute its affinity matrix $W$ by Eq.1, use the manifold ranking method to detect their own edge superpixels, and obtain boundaries ILM and IS/CL by fitting.

Step 2. Detect the other weak boundaries

Step 2.1 Align the filtered image according to the boundary IS/CL.

Step 2.2 Detect the low and middle contrast edge superpixels by canny edge detector for aligned image.

Step 2.3 Construct nine weight graphs $G_{5}, G_{4}, G_{6}, G_{2}, G_{3}, G_{7}, G_{9}, G_{10}$ and $G_{11}$ with edge superpixels as nodes, successively, compute their own affinity matrix $W$ by Eq.4, and utilize manifold ranking method to extract their own edge superpixels, and obtain their own boundaries by fitting.

Output: The lay segmentation 3D image.

\section{Experiments and Results}

We evaluated the proposed algorithm on two different labeled macular OCT datasets (Cirrus, Zeiss Meditec). All the 2D OCT slices were scanned from $6 \times 2 \times 6 \mathrm{~mm}^{3}$ around macula-centered 3D OCT volumes, and their main boundaries were manually traced by two independent observers (retinal specialists) with the use of a computer-aided manual segmentation procedure, and they marked the boundaries as ground truths. The first dataset contains 55 slices, which are from 11 different normal human's eyes, and each eye randomly selected 5 slices, respectively, there are seven $19 \times 496 \times 1024$ and four $19 \times$ 
$496 \times 512$ voxels. The second dataset contains 60 slices, is from 12 different abnormal human's eyes with CSR. Each eye randomly selected 5 slices whose sizes are $128 \times 200 \times$ 300 voxels, and their resolutions are much lower.

The proposed algorithm mainly compared with the two independent observers, the SLIC superpixels method [17], and the Iowa's algorithm [11] by the mean signed and unsigned border positioning differences of the ILM, NFL/GCL, IPL/INL, INL/OPL, OPL/ONL, ELM, IS/CL and BM/Choroid boundaries, since the two independent observers did not attempt to trace the GCL/IPL, CL/OS and OS/BM boundaries that are considered invisible. In addition, for the purpose of the clinical and medical analysis, the thickness map of each layer was respectively plotted by the proposed algorithm in the dataset 2 .

In a personal computer (CPU: Core 2, 2.53GHz, RAM: $4 \mathrm{~GB}$ ), the proposed algorithm was implemented in Matlab 2012b, and successfully detected all eleven intra-retinal boundaries in the two different datasets. For the full ten layers segmentation of each 2D slice, the proposed algorithm took about 5.28s much faster than 9.02s [11] and 9.6s [17] in dataset1, and the proposed algorithm also took about $2.9 \mathrm{~s}$ much faster in dataset 2 . For the mean signed and unsigned border positioning differences of the two datasets, Tabels $1,2,3$ and 4 respectively summarize the main boundaries as follows.

Table1. Unsigned border position differences (mean \pm SD in pixel) in Dataset1

\begin{tabular}{|c|c|c|c|c|}
\hline \multirow{2}{*}{ Border } & \multicolumn{4}{|c|}{ Segmenter } \\
\cline { 2 - 5 } & $\begin{array}{c}\text { Obs.1vs. } \\
\text { Obs.2 }\end{array}$ & $\begin{array}{c}\text { Alo_Iowa.vs. } \\
\text { Avg. Obs }\end{array}$ & $\begin{array}{c}\text { Alo_SLIC. } \\
\text { vs.Avg. Obs }\end{array}$ & $\begin{array}{c}\text { Alo_Proposed. } \\
\text { vs. Avg.Obs. }\end{array}$ \\
\hline ILM & $1.16 \pm 0.35$ & $2.70 \pm 1.15$ & $0.80 \pm 0.19$ & $0.78 \pm 0.29$ \\
\hline NFL/GCL & $1.88 \pm 0.64$ & $3.96 \pm 1.62$ & $1.18 \pm 0.64$ & $1.06 \pm 0.41$ \\
\hline IPL/INL & $1.47 \pm 0.39$ & $3.41 \pm 1.80$ & $1.06 \pm 0.49$ & $0.94 \pm 0.35$ \\
\hline INL/OPL & $1.56 \pm 0.40$ & $2.65 \pm 1.00$ & $0.94 \pm 0.32$ & $1.02 \pm 0.36$ \\
\hline OPL/ONL & $1.80 \pm 0.56$ & $2.46 \pm 0.69$ & $1.13 \pm 0.57$ & $1.13 \pm 0.46$ \\
\hline ELM & $1.14 \pm 0.42$ & --- & $0.98 \pm 0.45$ & $1.31 \pm 0.66$ \\
\hline IS/CL & $1.03 \pm 0.24$ & $1.33 \pm 0.38$ & $0.72 \pm 0.52$ & $0.65 \pm 0.22$ \\
\hline BM/Choroid & $1.51 \pm 0.54$ & $1.70 \pm 0.38$ & $0.90 \pm 0.25$ & $0.90 \pm 0.28$ \\
\hline Overall & $1.44 \pm 0.13$ & $2.60 \pm 0.57$ & $0.96 \pm 0.16$ & $0.97 \pm 0.14$ \\
\hline
\end{tabular}

For the dataset 1 , the Table 1 demonstrates that all the mean unsigned errors between the proposed algorithm vs. the reference standard are less than 1.31 pixels, are closed to the two independent observers, are similar with the SLIC superpixels algorithm, particularly, and less than the Iowa algorithm vs. the reference standard. In Table 2, for the mean signed border positioning differences, all the errors computed between the proposed algorithm vs. the reference standard are so much better than \pm 0.41 pixels and the Iowa algorithm vs. the reference standard. Particularly, the overall error -0.02 pixels approximates to zeros.

Table 2. Signed border position differences (mean $\pm S D$ in pixel) in Dataset 1

\begin{tabular}{|c|c|c|c|c|}
\hline \multirow{2}{*}{ Border } & \multicolumn{4}{|c|}{ Segmenter } \\
\cline { 2 - 5 } & $\begin{array}{c}\text { Obs.1vs. } \\
\text { Obs.2 }\end{array}$ & $\begin{array}{c}\text { Alo_Iowa.vs. } \\
\text { Avg. Obs }\end{array}$ & $\begin{array}{c}\text { Alo_SLIC. } \\
\text { vs.Avg. Obs }\end{array}$ & $\begin{array}{c}\text { Alo_Proposed. } \\
\text { vs. Avg.Obs. }\end{array}$ \\
\hline ILM & $-0.07 \pm 0.80$ & $-2.43 \pm 1.32$ & $-0.38 \pm 0.33$ & $-0.37 \pm 0.40$ \\
\hline NFL/GCL & $-1.65 \pm 0.81$ & $0.82 \pm 2.19$ & $0.05 \pm 0.52$ & $0.06 \pm 0.42$ \\
\hline IPL/INL & $-0.60 \pm 0.73$ & $1.40 \pm 2.16$ & $0.28 \pm 0.62$ & $0.32 \pm 0.43$ \\
\hline INL/OPL & $-0.61 \pm 0.76$ & $0.66 \pm 1.41$ & $-0.15 \pm 0.56$ & $-0.20 \pm 0.60$ \\
\hline
\end{tabular}




\begin{tabular}{|c|c|c|c|c|}
\hline OPL/ONL & $-0.23 \pm 0.98$ & $0.25 \pm 1.46$ & $0.07 \pm 0.50$ & $0.13 \pm 0.58$ \\
\hline ELM & $0.32 \pm 0.79$ & --- & $0.57 \pm 0.57$ & $-0.16 \pm 1.14$ \\
\hline IS/CL & $-0.21 \pm 0.61$ & $-1.22 \pm 0.46$ & $-0.30 \pm 0.65$ & $-0.33 \pm 0.41$ \\
\hline BM/Choroid & $-0.91 \pm 0.59$ & $-1.56 \pm 0.44$ & $0.44 \pm 0.30$ & $0.41 \pm 0.34$ \\
\hline Overall & $-0.49 \pm 0.13$ & $-0.30 \pm 0.71$ & $0.07 \pm 0.13$ & $-0.02 \pm 0.26$ \\
\hline
\end{tabular}

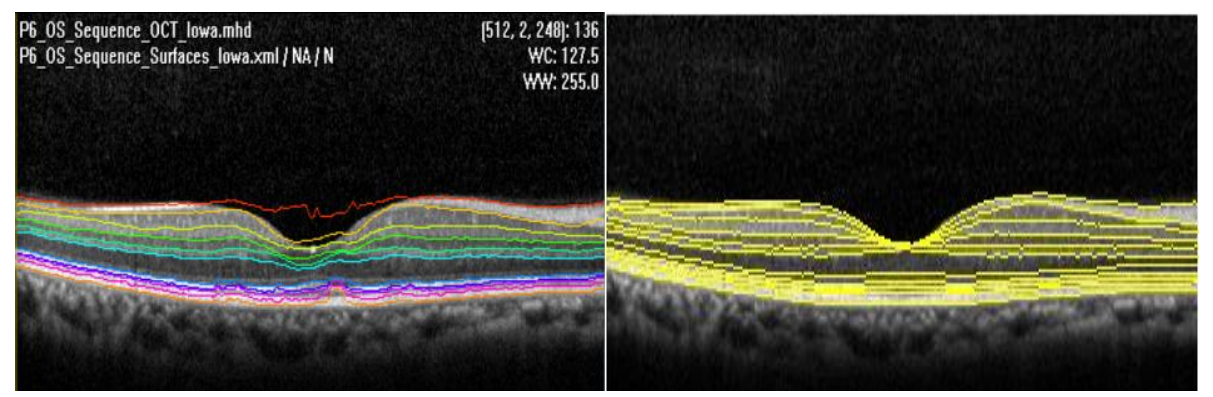

Figure 5. Comparison of the lowa algorithm (Left ) vs. the proposed algorithm (Right) segmentation on OCT image

Hereon, obviously, it is shown as Fig.5 that the detections of the proposed algorithm were much better than the detections of the Iowa algorithm around the macular fovea, which makes it difficult to detect some boundaries since their contrasts are much lower. For seven OCT image with $19 \times 496 \times 1024$ voxels, Fig.6 further illustrates that the unsigned border positioning errors of the main boundaries corresponding to every column, these errors computed between the proposed algorithm and the reference standard were less than those computed between the method of the Iowa and the reference standard. Both the Fig.5 and Fig. 6 also well demonstrate the reasons that the proposed algorithm was much better than the method of Iowa in Tables 1 and 2.
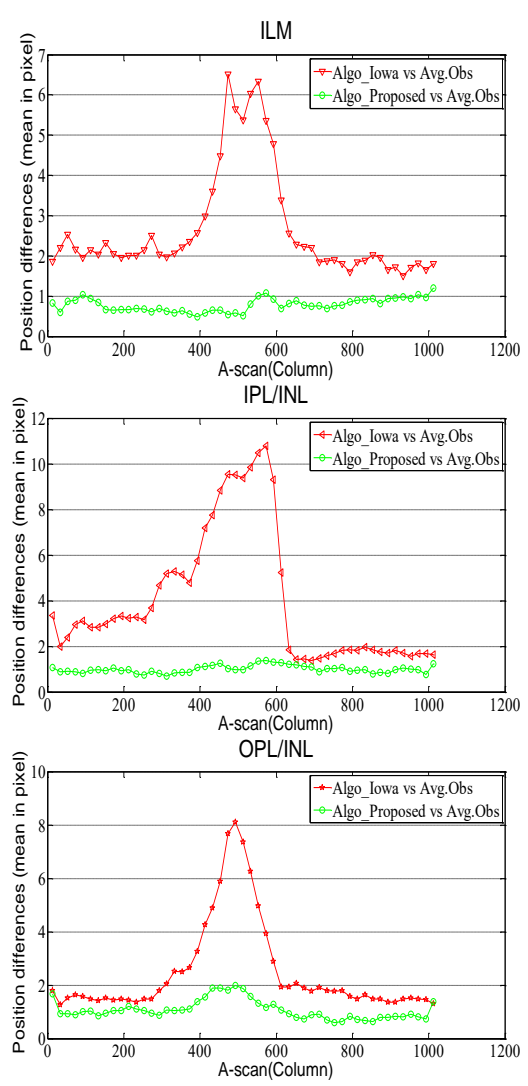
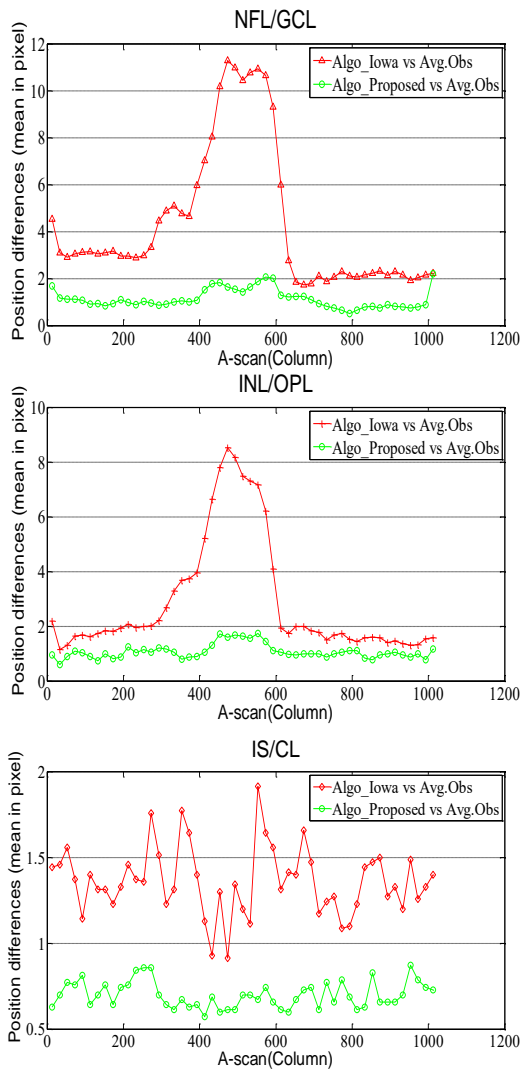

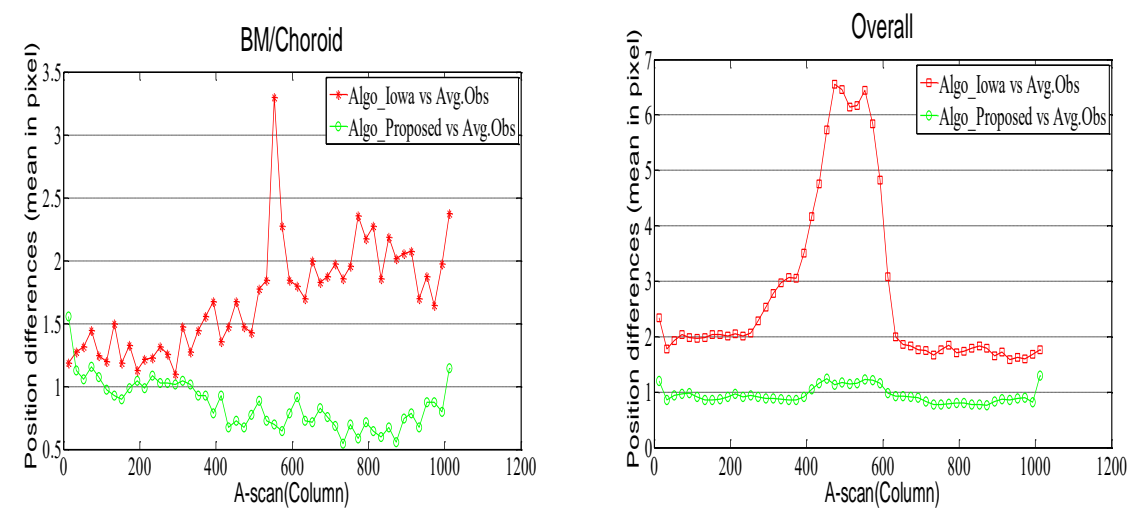

Figure 6. The Error Result Plots Show the Border Position Differences of the Main Seven Boundaries between the proposed Algorithm vs. the lowa Algorithm in Dataset 1
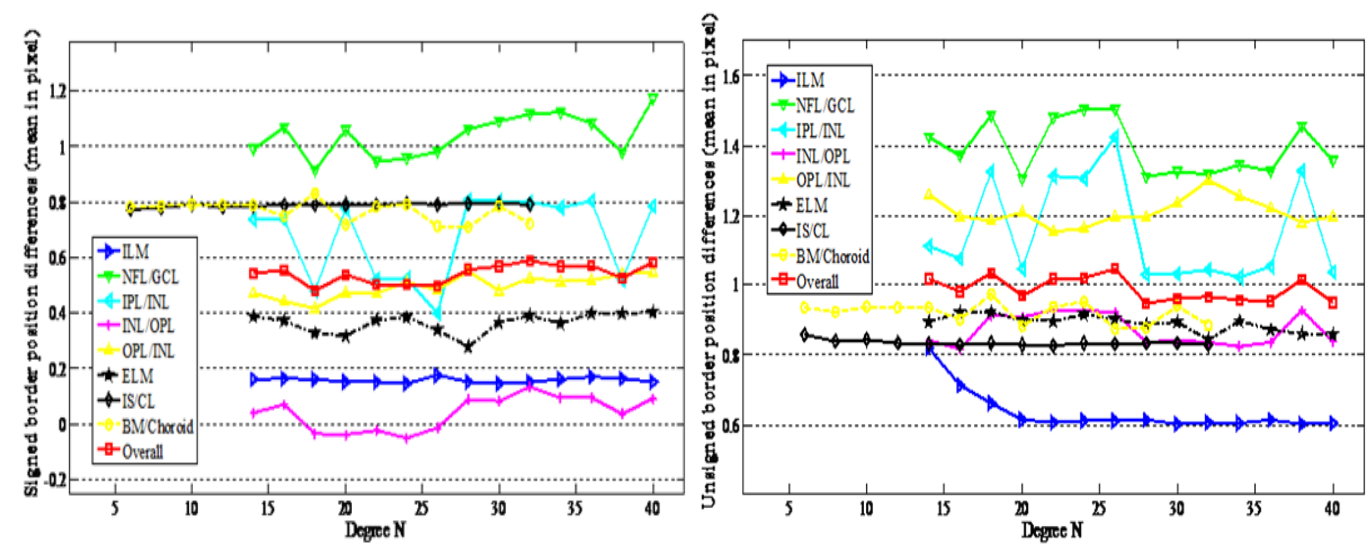

Figure 7. The Error Result Plots Show the Border Position Differences of the Main Eight Boundaries between the proposed Algorithm and the

Reference Standard in the dataset 2, when the degree $\mathbf{N}$ of the Polynomial Curve Fitting is set from 6 to 32 or 14 to 40 . Left: the Signed Border Position Differences. Right: the Unsigned Border Position Differences

For the degree $N$ of the polynomial curve fitting, respectively, we computed the signed and unsigned border position errors of the main eight boundaries between the proposed algorithm and the reference standard as shown in Figure 7. The result plot shows that the signed and unsigned border positioning errors of the overall and most fitting boundaries are only small fluctuations when the degree $N$ of the polynomial curve fitting is set from 6 to 32 or 14 to 40 . Therefore, the result plots suggest that the edge superpixels of the most boundaries are always continuously and perfectly extracted by our proposed algorithm. Certainly, for the relative larger errors fluctuations of the boundaries 2 and 4, it is possible that low contrast in local region results in the discontinuous edge superpixels, such as the edge superpixels of the boundaries 2 in Figure 3i, or personal errors between the proposed algorithm and the reference standard. The Tabels 1, 2 and Figure 7 sufficiently demonstrate that the proposed algorithm can not only effectively detect main boundaries, but also take on a good robustness around the macular fovea. 

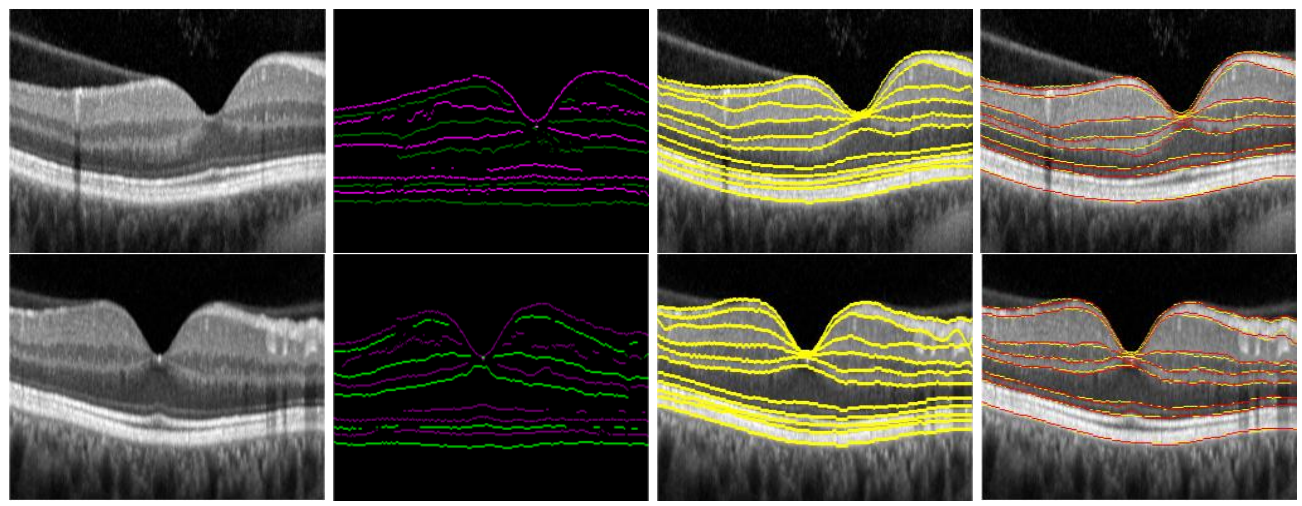

Figure 8. Comparison of Automatic (yellow) versus the Reference Standard (red) segmentation on images with blood vessel artifacts. (column a) Original image.( column b) Detected the edge superpixels with our proposed automatic method. (column c) Final segmentation with our proposed automatic method after smoothing. (column d) Comparison of automatic (yellow) versus the reference standard (red)

It is possible presence of organic texture artifacts in OCT image, for example, in the upper left or upper right corner in Figure 8a, which easily joint with ILM boundary, and caused inaccuracy in the detection of ILM boundary. Besides, the blood vessels generally result in discontinuities in macular OCT images, and it is inevitable challenge for automated segmentation thickness map generation [25]. The Figure 8 demonstrates that the proposed algorithm could effectively overcome organic texture artifacts and the blood vessel discontinuity problem, and improve the accuracy of the detection of boundaries. Because the proposed algorithm designed a simple adaptive filter, and an affinity matrix was incorporated neighboring information based on the edge superpixels of the graph.

For the macula 3D OCT images with CSR, which seriously affect the boundary detection, and become challenging due to the degradation of image quality and some additional structures [4, 12]. Tabel 3 and 4 show that the mean unsigned border positioning differences and the mean signed border position differences in the dataset 2 between the two independent observers vs. the reference standard, and the proposed algorithm vs. the reference standard. For the mean unsigned border positioning differences, Tabel 3 illustrates that all the mean errors between the proposed algorithm vs. the reference standard were less than 1 pixel, and were closed to the two independent observers. In Tabel 4, for the mean signed border positioning differences, all the errors computed between the proposed algorithm vs. the reference standard all so much better than \pm 0.34 pixels, and were closed to those errors between the two independent observers vs. the reference standard. Particularly, though the boundaries NFL/GCL and $\mathrm{BM} /$ Choroid are affected, the errors of the proposed algorithm also suggest that the proposed algorithm can also effectively detect main boundaries of macula 3D OCT images with CSR.

Table 3. Unsigned Border Position Differences (mean \pm SD in pixel) in Dataset2

\begin{tabular}{|c|c|c|c|c|}
\hline \multirow{2}{*}{ Border } & \multicolumn{4}{|c|}{ Segmenter } \\
\cline { 2 - 5 } & $\begin{array}{c}\text { Obs.1vs. } \\
\text { Obs.2 }\end{array}$ & $\begin{array}{c}\text { Alo_Proposed. } \\
\text { vs. Obs.1 }\end{array}$ & $\begin{array}{c}\text { Alo_Proposed. } \\
\text { vs. Obs.2 }\end{array}$ & $\begin{array}{c}\text { Alo_Proposed. } \\
\text { vs. Avg.Obs. }\end{array}$ \\
\hline ILM & $0.54 \pm 0.15$ & $0.51 \pm 0.11$ & $0.52 \pm 0.15$ & $0.49 \pm 0.08$ \\
\hline NFL/GCL & $0.59 \pm 0.21$ & $0.82 \pm 0.20$ & $0.84 \pm 0.24$ & $0.80 \pm 0.18$ \\
\hline IPL/INL & $0.64 \pm 0.24$ & $0.73 \pm 0.24$ & $0.73 \pm 0.21$ & $0.69 \pm 0.20$ \\
\hline INL/OPL & $0.57 \pm 0.20$ & $0.64 \pm 0.18$ & $0.70 \pm 0.19$ & $0.64 \pm 0.15$ \\
\hline
\end{tabular}




\begin{tabular}{|c|c|c|c|c|}
\hline OPL/ONL & $0.59 \pm 0.21$ & $0.63 \pm 0.16$ & $0.61 \pm 0.15$ & $0.59 \pm 0.12$ \\
\hline ELM & $0.56 \pm 0.20$ & $' 0.62 \pm 0.20$ & $0.70 \pm 0.20$ & $0.64 \pm 0.15$ \\
\hline IS/CL & $0.52 \pm 0.17$ & $0.47 \pm 0.12$ & $0.47 \pm 0.17$ & $0.45 \pm 0.10$ \\
\hline BM/Choroid & $0.66 \pm 0.24$ & $0.91 \pm 0.31$ & $0.93 \pm 0.37$ & $0.88 \pm 0.33$ \\
\hline Overall & $0.58 \pm 0.03$ & $0.67 \pm 0.06$ & $' 0.69 \pm 0.07$ & $0.65 \pm 0.08$ \\
\hline
\end{tabular}

Table 4. Signed Border Position Differences (mean \pm SD in pixel) in Dataset 2

\begin{tabular}{|c|c|c|c|c|}
\hline \multirow{2}{*}{ Border } & \multicolumn{4}{|c|}{ Segmenter } \\
\cline { 2 - 5 } & $\begin{array}{c}\text { Obs.1vs. } \\
\text { Obs.2 }\end{array}$ & $\begin{array}{c}\text { Alo_Proposed. } \\
\text { vs. Obs.1 }\end{array}$ & $\begin{array}{c}\text { Alo_Proposed. } \\
\text { vs. Obs.2 }\end{array}$ & $\begin{array}{c}\text { Alo_Proposed. } \\
\text { vs. Avg.Obs. }\end{array}$ \\
\hline ILM & $-0.03 \pm 0.34$ & $0.27 \pm 0.21$ & $-0.24 \pm 0.25$ & $0.26 \pm 0.15$ \\
\hline NFL/GCL & $0.03 \pm 0.35$ & $-0.01 \pm 0.39$ & $0.02 \pm 0.38$ & $0.01 \pm 0.34$ \\
\hline IPL/INL & $0.22 \pm 0.34$ & $-0.34 \pm 0.25$ & $-0.12 \pm 0.34$ & $-0.23 \pm 0.24$ \\
\hline INL/OPL & $0.17 \pm 0.30$ & $0.01 \pm 0.32$ & $0.18 \pm 0.30$ & $0.09 \pm 0.27$ \\
\hline OPL/ONL & $-0.00 \pm 0.40$ & $-0.21 \pm 0.26$ & $-0.21 \pm 0.30$ & $-0.21 \pm 0.20$ \\
\hline ELM & $0.18 \pm 0.37$ & $0.06 \pm 0.54$ & $0.25 \pm 0.59$ & $0.15 \pm 0.53$ \\
\hline IS/CL & $0.08 \pm 0.33$ & $-0.18 \pm 0.23$ & $-0.10 \pm 0.22$ & $-0.14 \pm 0.16$ \\
\hline BM/Choroid & $0.11 \pm 0.49$ & $-0.16 \pm 0.54$ & $-0.05 \pm 0.63$ & $-0.11 \pm 0.53$ \\
\hline Overall & $0.09 \pm 0.06$ & $-0.07 \pm 0.13$ & $0.03 \pm 0.15$ & $-0.02 \pm 0.15$ \\
\hline
\end{tabular}

Figure 9 also shows a typical segmentation results for a three-dimensional volume of a CSR eye from dataset2, and illustrates the corresponding segmented 3D surfaces for the ILM, NFL/GCL, IPL/INL, IS/CL and BM/Choroid, and the corresponding 2D thickness maps for the NFL, ganglion cell complex (GCC: from 2nd to 4th boundary), OPL, $\mathrm{CL}+\mathrm{OS}$ and the total retina.

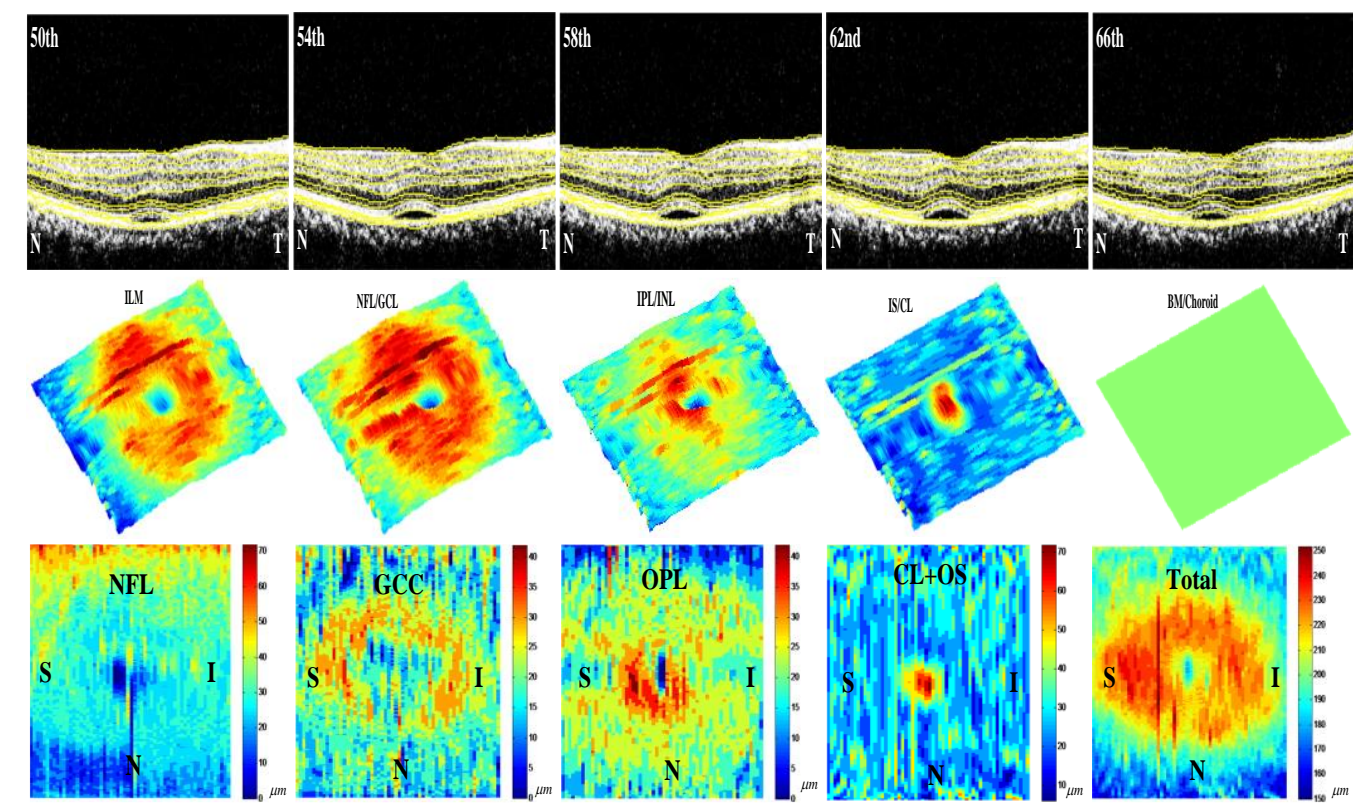

Figrue 9. Results of a CSR 3D vertical scan volume. (row a) five B-scans (the 50th, 54th, 58th, 62nd, 66th and 70th images) of this volume. (row b)

The segmented surfaces of 3D OCT image (surface 1 ILM, surface 2 NFL/GCL, surface 4 IPL/INL, surface 8 IS/CL, and surface $11 \mathrm{BM} /$ Choroid). (row c) NFL, GCC, OPL, CL+OS, and total retinal thickness maps covered $6 \times 6 \mathrm{~mm}^{2}$ area 


\section{Conclusion}

Inspired by superpixels, this paper well defined the edge superpixels, designed an adaptive filter, and proposed a graph-based manifold ranking method to segment macular retinal layers in 3D OCT images. It made the edge superpixel to substitute for single pixel, and considered the edge superpixels as nodes, which incorporated gradient cues and spatial priors of the edge superpixels. Based on the gradient sum and spatial distance of the edge superpixels, we utilized graph-based manifold ranking approach to extract corresponding boundaries. By testing a normal macular 3D OCT dataset and a macular 3D OCT dataset with CSR, We evaluated the proposed algorithm on main boundaries error. The experiments demonstrated promising results with comparisons to the manual tracings of two independent observers and the method of the [11, 17]. The proposed algorithm can not only overcome the organic texture artifacts and speckle noise, but also be computationally efficient, and be not relatively susceptible to the low contrast of macular fovea and the bad structure of the CSR. Therefore, the proposed algorithm is a fast, effective and robust layer segmentation method of macular OCT images. The future work will focus on the segmentation and thickness analysis of retinal layers in OCT images with ocular disease problems.

\section{Acknowledgments}

This work was supported in part by the Natural Science Foundation of China under Grant 61472102, and 61672194, by the Shandong Provincial Natural Science Foundation, China under Grant ZR2016FM04, and by the Humanity and Social Science Youth foundation of Ministry of Education of China under Grant 14YJC760001.

\section{References}

[1] Huang D., Swanson E. A., Lin C. P., Schuman J. S., Stinson W. G., Chang W., Hee M. R., Flotte T., Gregory K., Puliafito C. A. and Fujimoto J. G., "Optical coherence tomography", Science, vol. 254, no.5035, (1991), pp.1178-1181.

[2] Drexler, W. \& Fujimoto, JG., "State-of-the-art retinal optical coherence tomography", Prog. Retin. Eye Res. vol. 27, no.1, (2008), pp. 45-88.

[3] R.K. Murthy, Shamim Haji, Kumar Sambhav, Sandeep Grover, K.V. Chalam, "Clinical applications of spectral domain optical coherence tomography in retinal diseases", Biomedical Journal, vol.39, no.2, (2016), pp.107-120.

[4] Wani J. S., Bhat P. A., Ahangar A., et al. "Role of Optical Coherence Tomography in Central Serous Chorioretinopathy", Journal of Evolution of Medical and Dental Sciences, vol. 45, no. 4, (2015), pp. 7801-7809.

[5] Jean-Claude Mwanza, Donald L. Budenz, David G. Godfrey, Arvind Neelakantan, Fouad E. Sayyad, Robert T. Chang, Richard K. Lee, "Diagnostic Performance of Optical Coherence Tomography Ganglion Cell-Inner Plexiform Layer Thickness Measurements in Early Glaucoma”, Ophthalmology, vol.121, no.4, (2014), pp.849-854.

[6] D. Koozekanani, K. Boyer, C. Roberts, "Retinal thickness measurements from optical coherence tomography using a Markov boundary model”, IEEE Trans. Med. Imag., vol.20, no.9, (2001), pp.900916.

[7] A. Mishra, A. Wong, K. Bizheva, and D. A. Clausi, "Intra-retinal layer segmentation in optical coherence tomography images", Opt. Exp. vol.17, no.26, (2009), pp.23719-23728.

[8] MA. Mayer, R. P. Tornow, R. Bock, J. Hornegger and F. E. Kruse, "Automatic Nerve Fiber Layer Segmentation and Geometry Correction on Spectral Domain OCT Images Using Fuzzy C-Means Clustering", Invest. Ophthalmol. Vis. Sci., vol. 49, (2008), pp.1880.

[9] K. A. Vermeer, J. van der Schoot, H. G. Lemij, and J. F. de Boer, "Automated segmentation by pixel classification of retinal layers in ophthalmic OCT images", Biomed. Opt. Exp., vol.2, no.6, (2011), pp.1743-1756.

[10] Haeker, M., Abràmoff, M., Kardon, R., Sonka, M., "Segmentation of the surfaces of the retinal layer from OCT images”, Med Image Comput Comput Assist Interv, vol.9, no.1, (2006), pp.800-807.

[11] M.K. Garvin, M.D. Abramoff, "Automated 3-D Intraretinal Layer Segmentation of Macular pectralDomain Optical Coherence Tomography Images", IEEE Transaction on Medical Imaging, vol.28, no.9, (2009), pp.1436-1447. 
[12] Shi Fei, Chen Xinjian, Zhao Heming, Zhu Weifang, Xiang Dehui, Gao Enting, Sonka M. and Chen Haoyu. "Automated 3-D retinal layer segmentation of macular optical coherence tomography images with serous pigment epithelial detachments", IEEE Trans Med. Ima. vol.34, no.2, (2015), pp.441 - 452.

[13] SJ. Chiu, XT .Li, P. Nicholas, CA. Toth, JA. Izatt, and S. Farsiu, "Automatic segmentation of seven retinal layers in SDOCT images congruent with expert manual segmentation", Optic Express, vol.18, no.18, (2010), pp.19413-19428.

[14] Q. Yang, CA. Reisman, Z. Wang, Y. Fukuma, M. Hangai, N. Yoshimura, A. Tomidokoro, M. Araie, AR. Raza, DC. Hood and K. Chan, "Automated layer segmentation of macular OCT images using dualscale gradient information”, Optics Express. vol.18, no.20, (2010), pp.21294-21307.

[15] Y. M. Cha, J.H. Han, "High-Accuracy Retinal Layer Segmentation for Optical Coherence Tomography Using Tracking Kernels Based on Gaussian Mixture Model", IEEE Journal of Selected Topics in Quantum Electronics, vol.20, no.2, (2014), pp.1743-1756.

[16] R Kafieh, H Rabbani, MD Abramoff, M Sonka, "Intra-retinal layer segmentation of 3D optical coherence tomography using coarse grained diffusion map", Medical image analysis, vol.17, no.8, (2013), pp. 907-928.

[17] Zhijun Gao, Wei Bu, Yalin Zheng, and Xiangqian Wu, "Automated layer segmentation of macular OCT images via Graph-Based SLIC Superpixels and Manifold Ranking Approach", Computerized Medical Imaging and Graphics, (2016), DOI information: 10.1016/j.compmedimag.2016.07.006.

[18] J.J. Yan, Y.N. Yu, X.Y. Zhu, Z. Lei, S. Z. Li, “Object Detection by Labeling Superpixels”, In CVPR, (2015) June 5107-5116.

[19] C. Yang and L. H. Zhang, H. C. Lu, et al, "Saliency Detection via Graph-Based Manifold Ranking," In CVPR, (2013) June 3166-3173.

[20] HA Kierstead, "Applications of edge coloring of multigraphs to vertex coloring of graphs," Discrete Mathematics", vol.74, no.1, (1989), pp.117-124.

[21] Canny, John, "A Computational Approach to Edge Detection", IEEE Transactions on Pattern Analysis and Machine Intelligence, vol.8, no.6, (1986), pp.679-698.

[22] D. Zhou, J. Weston, A. Gretton, O. Bousquet, and B. Scholkopf, "Ranking on data manifolds", In NIPS, (2004), pp.169-176.

[23] B. Scholkopf, J. Platt, J. Shawe-Taylor, A. Smola, and R. Williamson, "Estimating the support of a highdimensional distribution", Neural Computation, vol.13, no.7, (2001), pp.1443-1471.

[24] A. Ng, M. Jordan, Y. Weiss, et al, "On spectral clustering: Analysis and an algorithm”, In NIPS, (2002), pp.849-856.

[25] D. C. Hood, B. Fortune, S. N. Arthur, D. Xing, J. A. Salant, R. Ritch, and J. M. Liebmann, "Blood vessel contributions to retinal nerve fiber layer thickness profiles measured with optical coherence tomography”, J. Glaucoma, vol.17, no. 7, (2008), pp.519-528.
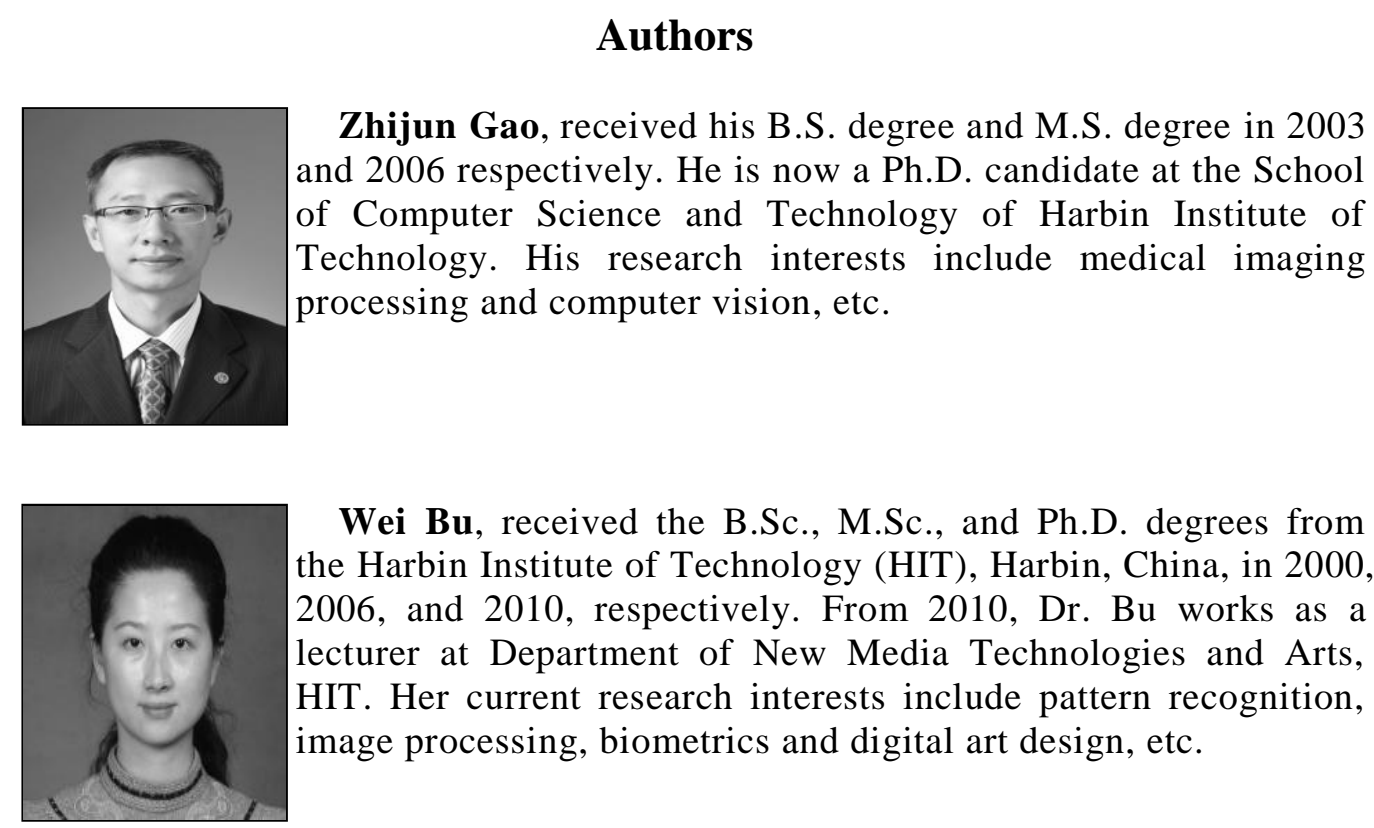

Wei Bu, received the B.Sc., M.Sc., and Ph.D. degrees from the Harbin Institute of Technology (HIT), Harbin, China, in 2000, 2006 , and 2010, respectively. From 2010, Dr. Bu works as a lecturer at Department of New Media Technologies and Arts, HIT. Her current research interests include pattern recognition, image processing, biometrics and digital art design, etc. 


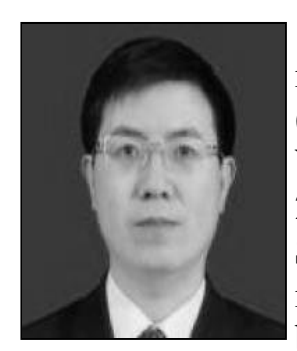

Xiangqian Wu, received the B.Sc., M.Sc., and Ph.D. degrees in computer science from the Harbin Institute of Technology (HIT), Harbin, China, in 1997, 1999, and 2004, respectively. Dr. Wu works as a lecturer (2004-2006), associate professor (20062009) and professor (2009-present) at the School of Computer Science and Technology, HIT. His current research interests include pattern recognition, image processing, computer vision, biometrics and medical image analysis, etc.

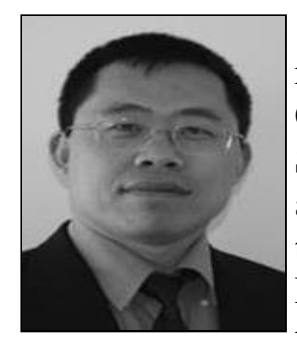

Yalin Zheng, received his BEng and MEng in 1994 and 1997 respectively, and received his $\mathrm{PhD}$ in Image Processing and Computer Vision from the School of Electronics and Computer Science, University of Southampton in 2003. Dr. Zheng works as an Associate Professor/Senior Lecturer in Ophthalmic Imaging at the Department of Eye and Vision Science, University of Liverpool. His current research interests include image processing, computer vision, data mining and medical imaging analysis. 
International Journal of Future Generation Communication and Networking Vol.10, No.6 (2017) 